Einführung in das Schwerpunktthema

\title{
Nachhaltig Investieren
}

G

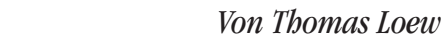
rünes Geld hieß vor vier Jahren die erste Studie des Instituts für ökologische Wirtschaftsforschung, die sich mit interessensgeleiteten Geldanlagen beschäftigt hat (1). Die Studie und die gleichnamige Messe fanden damals enorme Resonanz in den Medien. Der Begriff hat sich inzwischen gewandelt: statt vom Grünen Geld spricht man heute von ökologisch-ethischen Geldanlagen und nachhaltigem Investieren, ohne dass sich bislang ein einheitlicher Begriff im deutschsprachigen Raum durchgesetzt hat (2). International hat sich die Fachwelt dagegen auf socially responsible investment, kurz SRI, geeinigt.

Welchen Begriff man auch wählt, der Markt boomt in Deutschland wie auch international. Beispielsweise ist die Zahl der in Deutschland angebotenen ökologisch-ethischen Investmentfonds von 12 im Jahre 1998 auf aktuell 50 angestiegen (vgl. auch den Beitrag von Bartolomeo und Wilhelm). Europaweit werden rund 280 SRI-Fonds mit einem Volumen von rund 14,4 Billionen Euro aufgelegt (3). Immer noch handelt es sich um einen kleinen Nischenmarkt, der sich in Größenordnungen unter zwei Prozent des jeweiligen Gesamtmarkts bewegt. Allerdings wird davon ausgegangen, dass sich das bisherige dynamische Wachstum weiter fortsetzt. Ökologisch-ethisches Investieren gewinnt international auch bei Versicherungen, Pensionsfonds und anderen institutionellen Anlegern zunehmend an Bedeutung.

Hintergrund der positiven Entwicklung ist auch ein Wandel der Motivation und der Perspektiven. Am Anfang standen in den siebziger Jahren Anleger, die ihre Ersparnisse entsprechend ihrer persönlichen Werte anlegen wollten. Dies führte in Deutschland unter anderem zur Gründung der GLS-Bank, der Ökobank und des Finanzberaters Versiko. Die beiden Banken entwickelten für ihre Kunden Anlageformen, mit denen in der Regel gezielt bestimmte soziale oder ökologische Projekte und Unternehmen finanziert wurden.

Während damals die Mehrheit der moralisch motivierten Anleger bewusst auch auf eine Maximierung ihrer Rendite verzichtete, hat sich inzwischen gezeigt, dass durch eine ökologisch-ethische Anlagepolitik bei der Aktienauswahl auch die Rentabilität gesteigert werden kann. Den Nachweis brachte erstmals der Dow-Jones-Sustainability-Index, der längere Zeit eine bessere Leistung als sein Referenzindex zeigen konnte (siehe Abbildung 1). Vor diesem Hintergrund strebt beispielsweise auch der zweitgrößte europäische Pensionsfonds PGGM, der in den Niederlanden ansässig ist, mittelfristig an, dass seine gesamten Anlagen unter Berïcksichtigung von Nachhaltigkeitsaspekten verwaltet werden. Vorstandsmitglied Roderick Munsters geht davon aus, dass letztendlich alle institutionellen Investoren nachhaltig anlegen werden (4). Innerhalb der deutschen Banken spiegelt die Umweltbank oder auch die kürzlich gegründete Ethikbank die Verbindung zwischen gutem Gewissen und Rendite wider.

\section{- Kritische Fragen}

Bei der Freude an der positiven Entwicklung scheint sich bislang kaum jemand zu trauen, auch kritische Fragen zu stellen. Betrachtet man die Entwicklung des inzwischen berühmten Dow Jones Sustainability Indexes genauer, dann stellt man fest, dass er etwa seit Januar 2000 eine schlechtere Entwicklung aufweist als sein Referenzindex. Auch dem später aufgelegten FTSE4Good ergeht es in dieser Hinsicht nicht besser. Dennoch fließen weiter Mittel in die SRI-Fonds und die neu aufgelegten Indexprodukte. Die Anleger haben offensichtlich die Hoffnung nicht aufgegeben und sind weiterhin davon überzeugt, dass mit einer guten Leistung in ökologischen und sozialen Fragen auch ein gutes Management und insbesondere eine auf langfristige Wertsteigerungen ausgerichtete Unternehmensstrategie einhergeht.
Die zweite kritische Frage nach der Wirksamkeit kann auf jeden Fall positiv beantwortet werden (5). $\mathrm{Zu}$ einer verbesserten Finanzierung für ökologische oder soziale Projekte bzw. Unternehmen führen insbesondere Direktinvestitionen, woraus sich in Deutschland die erfolgreiche Windkraftindustrie entwickelt hat. Wenn SRI-Investmentfonds oder Versicherungen mit einer SRI-Anlagepolitik in Aktien investieren, dann hat dies aufgrund der immer noch vergleichsweise geringen Volumina in der Regel bislang keinen nennenswerten Einfluss auf die Aktienkurse der Unternehmen, sodass sich daraus keine Refinanzierungsvorteile für die auserwählten Unternehmen ergeben. Bedeutend sind jedoch die indirekten Effekte. Die Aufnahme in einen SRI-Index oder das gute Abschneiden in einem SRI-Rating gelten als Qualitätsmerkmale, die sich positiv auf das Image auswirken. Daher werden nicht nur die Fragen der Analysten gerne beantwortet und Nachhaltigkeitsberichte erstellt, sondern auch unternehmensintern die Verpflichtung zu Nachhaltigkeit deutlich gestärkt.

\section{Die Beiträge im Überblick}

Der Schwerpunkt Perspektiven nachhaltiger Finanzanlagen läßt viele Akteure, die durch ihre Arbeit zu der Entwicklung beitragen, zu Wort kommen.

Den Auftakt machen Matheo Bartolomeo und Axel Wilhelm, die die Trends in der Branche näher beleuchten und zu der Einschätzung kommen, dass in Europa die Strategie aktiven Engagements bei institutionellen Investoren noch deutliche Entwicklungspotenziale aufweist. Dies wird sich auch auf den Markt für SRI-Rating-Agenturen auswirken. Marnie Bammert gibt hier einen Überblick zu den verschiedenen Dienstleistungen, auf die die SRI-Vermögensverwalter zurückgreifen kön-
Abbildung 1: Dow Jones Sustainability Index im Leistungsvergleich

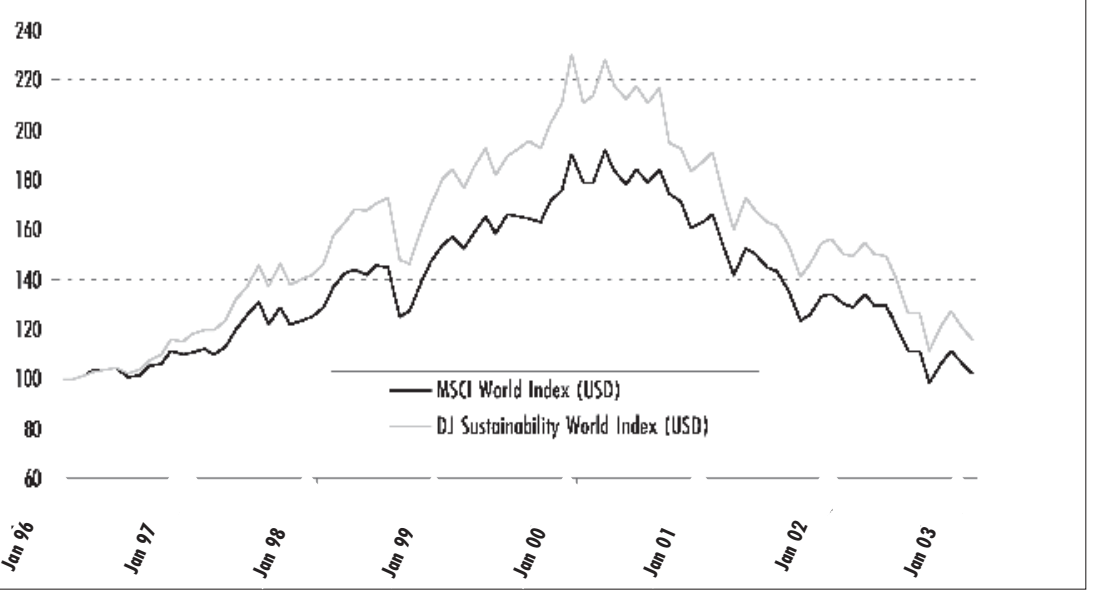

Quelle: SAM Sustainable Asset Management 
nen. Beide Artikel gehen auch auf die Entwicklung von Qualitätsstandards ein, in deren Entwicklung die verschiedenen Rating-Organisationen involviert sind. Hier lässt sich erkennen, dass das Qualitätsargument auch in dieser Branche an Bedeutung gewinnt.

Die Entwicklung des britischen SRI-Markts bekam einen starken Impuls durch die im Jahr 2000 in Kraft getretene Offenlegungspflicht, die britische Pensionsfonds verpflichtet ihre Versicherten darüber zu unterrichten, ob und in welcher Form sie ökologische oder ethische Aspekte in der Anlage der Versicherungsbeiträge berücksichtigen. Diese Regulierung hat international eine deutliche Vorbildfunktion entwickelt. Im Beitrag von Thomas Loew wird eine Übersicht zu den bestehenden Regulierungen zur Förderung von SRI-Märkten gegeben. Es zeigt sich, dass nicht überall von Erfolgen berichtet werden kann.

Daneben gibt es natürlich immer noch praktische Probleme, die für einen breiteren Absatz von ökologisch-ethischen Geldanlagen bewältigt werden müssen. Petra Thome zeigt auf, dass der Vertrieb von SRI-Investmentfonds eine zusätzliche Herausforderung an Kunden und Berater darstellt. Konventionelle Anlageberater kennen sich in der Regel nicht in dem neuen Fachgebiet aus. Für das Beratungsgespräch mit dem Anleger stellt SRI eine zusätzliche Fragestellung dar, die neben den klassischen Überlegungen erörtert werden muss. SRI bedeutet also für beide Seiten einen zusätzlichen Aufwand.

Für mehr Transparenz für Anleger und Berater will die neu eingerichtete Internetplattform www.nachhaltiges-investment.de sorgen. Hier wird unter anderem eine Datenbank mit den im deutschsprachigen Raum angebotenen SRI-Investmentfonds samt Hintergrundinformationen angeboten. $\mathrm{Pa}$ schen von Flotow, Rolf-D. Häßler, Christian Hochfeld und Kathrin Graulich berichten über die Plattform und erste Auswertungen, die auf dieser Basis vorgenommen wurden. An dieser Stelle sei auch auf das von imug durchgefuihrte Ranking von 40 SRI-Fonds verwiesen - ebenfalls ein Beitrag zu mehr Transparenz und zur Diskussion über die Anforderungen an SRI-Investmentfonds (6). Die Anbieter auf den SRI-Märkten haben inzwischen Verbände gegründet, um ihre Interessen in der Politik besser durchsetzen zu können und den Informationsaustausch zu fördern. In Deutschland hat diese Aufgabe das Forum für Nachbaltige Geldanlagen übernommen (7). Über die Aktivitäten des Europäischen Dachverbands Eurosif berichten Matt Christensen und Sylvain Guyoton.
Abschließend wird der Horizont ausgeweitet: Einen umweltpolitischen Blickwinkel nimmt der Beitrag von Ingeborg Schumacher-Hummel. Sie untersucht, welche Auswirkungen der Erfolg von SRI auf die Corporate Governance der Pensionskassen haben wird. Es zeigt sich, dass die Pensionskassen durchaus ein neuer Treiber in Richtung Nachhaltigkeit sein können, wenn sich der bislang identifizierte positive Zusammenhang zwischen SRI und Performance weiter bestätigt.

Den Schlusspunkt bildet eine kritische Betrachtung der globalen Finanzmärkte. Kurt Hübner weist auf die Probleme des dynamischen Wachstums der Finanztransaktionen hin. Folgen sind Währungskrisen, eine hohe Volatilität weltwirtschaftlicher Preise und ein zunehmend verkürzter Zeithorizont der Marktteilnehmer, Faktoren, die sich im Prinzip durchweg negativ auf die angestrebte Nachhaltigkeit auswirken. Dabei stellt sich auch die Frage, ob die ökologisch-ethisch motivierten Anleger nicht ihren ungewollten Anteil an dieser Entwicklung haben.

\section{Anmerkungen}

(1) Vgl. Öko-Zentrum Nordrhein-Westfalen (Hrsg.): Studie Grünes Geld. Hamm 1999, sowie Franck, Kirein: Grüne Dagoberts und edle Spender. In: Ökologisches Wirtschaften, Nr. 4/1999. S. 7.

(2) Vgl. Loew, Thomas: Internationale Entwicklung der Regulierungen zur Förderung ökologisch-ethischer Finanzdienstleistungen, Diskussionspapier des I0̈W 56/2002, Berlin. Download unter www.ioew.de/home/downloaddateien/DP5602.pdf

(3) Stand Ende 2001, Vgl. SIRI Group (Hrsg.): Green, social and ethical funds in Europe 2002; Download unter www.sricompass.org/uploadstore/cms/docs/SRI_green_ social_ethical_funds_2002.pdf.

(4) Vgl. Bergius, Susanne: Nachhaltig anlegen heift an Wert gewinnen. In: Handelsblatt vom 11. Juli 2002.

(5) Vgl. z.B. Kahlenborn, Walter: Nützen nachhaltige Geldanlagen? In: Ökologisches Wirtschaften Nr. 3-4/2001, S.

5, sowie Murphy, Andrew: Die Rolle der Investoren für den ökologischen Strukturwandel. In: Ökologisches Wirtschaften Nr. 5/2001, S. 20-21.

(6) Vgl. Grazek, Uwe: Eine Bewertung ökologisch-ethischer Investmentfonds. In: Ökologisches Wirtschaften Nr.

$1 / 2003$, S. 5.

(7) Vgl. www.Forum-NG.de

\section{Der Autor}

Thomas Loew ist wissenschaftlicher Mitarbeiter im Forschungsfeld ökologische Unternehmenspolitik des Instituts für ökologische Wirtschaftsforschung (IÖW).

Kontakt: IÖW, Potsdamer Str. 105, 10785 Berlin. Tel. 030-884594-17, E-Mail: Thomas.Loew@ioew.de

\section{politische ökologie ${ }^{77-78}$}

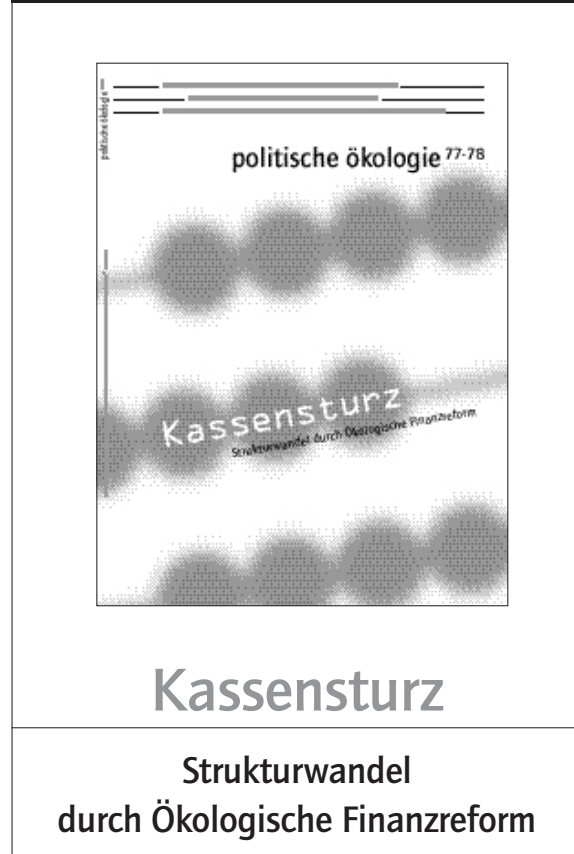

Wenns ums Geld geht, hört der Spaß auf! Jeder Eingriff in das traditionelle staatliche Finanzierungssystem ist sehr schwierig. Aber besonders eine Ökologische Finanzreform, die die Ökosteuern und die Kritik der konventionellen Subventionspolitik umfasst und die viele Strukturen umwälzen könnte, ist kurz vor der Wahl ein heißes Eisen. Trotzdem äußern sich in der aktuellen politischen ökologie prominente Politiker wie Hans Eichel und Jürgen Trittin zu diesen wichtigen Themen. Ferner haben sich Wirtschaftswissenschaftler, Akteure aus dem Umweltbereich und weitere kluge Köpfe ihre Gedanken gemacht.

Mit Beiträgen von J. Nill, A. Volkens K. Schlegelmilch, A. Görres, A. Burger, I. Hanhoff, K. Knickel u.v.a.

\section{www.oekom.de}

Abonnement von sechs Ausgaben für Institutionen: 95,00 € (+ Online-Abo 142,50 €) Einzelpersonen: $55,00 €$ (+ Online-Abo 82,50€) Studentenabo: $45,00 €$ (+ Online-Abo $67,50 €$ ) Einzelheft: $15,00 €$

Zu beziehen bei: CONSODATA ONE-TO-ONE

Semmelweisstraße 8, D-82152 Planegg

Fon $++49 /(0) 89 / 857$ 09-155

Fax $++49 /(0) 89 / 857$ 09-131

E-Mail kontakt@oekom.de 
(c) 20I0 Authors; licensee IÖW and oekom verlag. This is an article distributed under the terms of the Creative Commons Attribution Non-Commercial No Derivates License (http://creativecommons.org/licenses/by-nc-nd/3.o/), which permits unrestricted use, distribution, and reproduction in any medium, provided the original work is properly cited. 